\title{
Effect of Polysorbates on the Growth of Rhodotorula Glutinis in Oil Rich Medium
}

\author{
Svetlana RAITA ${ }^{1 *}$, Kriss SPALVINS ${ }^{2}$, Evalds RAITS ${ }^{3}$, Inese SILICKA ${ }^{4}$, \\ Dagnija BLUMBERGA ${ }^{5}$ \\ ${ }^{1,2,5}$ Riga Technical University, Institute of Energy Systems and Environment, Azenes street 12/1, LV-1048 \\ Riga, Latvia \\ ${ }^{3}$ Latvia University of Life Sciences and Technologies, Faculty of Food Technology, Riga street 22, \\ LV-3004, Jelgava, Latvia \\ ${ }^{4}$ Rezekne Academy of Technologies, Faculty of Economics and Management, Atbrivosanas aleja 115, \\ LV-4601, Rezekne, Latvia
}

\begin{abstract}
The present study has investigated the effect of oil rich medium supplementation with polysorbates Tween 20,40 and 80 , for the cultivation of red oleaginous yeast Rhodotorula glutinis. $R$. glutinis has been cultivated in yeast extract peptone glucose modified broth (mYPG) supplemented with $2 \%$ of waste cooking rapeseed oil and three polysorbate types with $0.5 \%, 1 \%, 2 \%, 3 \%, 4 \%, 5 \%, 6 \%, 7 \%, 8 \%, 9 \%$ and $10 \%$ concentration each. Yeast biomass was measured by the thermogravimetric method at $105^{\circ} \mathrm{C}$ each day during 7-day experiment. The oil rich medium supplementation with Tween 20,40 and 80 at concentrations ranging from $2 \%$ to $10 \%$ significantly increased the biomass of $R$. glutinis. All three types of the studied polysorbates with $0.5 \%$ and $1 \%$ concentration, did not affect yeast growth and the dry biomass - results were similar to the control sample without polysorbate addition. Between the three types of polysorbates, Tween 20 was selected as the preferable for $R$. glutinis cultivation with an optimal concentration of $2 \%$. Cultivation of $R$. glutinis in oil rich medium with polysorbates Tween 20,40 and 80, supplementation up to $10 \%$ concentration did not have had an inhibitory effect on the biomass growth.
\end{abstract}

Keywords - Dry biomass; oil emulsification; oleaginous yeast; Tween; waste cooking oil

\section{INTRODUCTION}

Since the last century, the ability of microorganisms to produce industrially important substances has been discovered and is still being actively studied. A large number of filamentous fungi, bacteria, microalgae and yeasts are used to obtain enzymes, lipids, vitamins, amino acids and antibiotics [1]-[3]. The particular interest of researchers was attracted by the oleaginous yeasts, such as Rhodosporidium toruloides [4], Trichosporon dermatis [5], Cryptococcus curvatus [6], Yarrowia lipolytica [7], Rhodotorula glutinis [8] for lipids, pigments and enzymes (lipase, cellulase, amylase, etc.) production.

$R$. glutinis is a promising oleaginous yeast that produces a significant amount of lipids and carotenoids (torulene, $\beta$-carotene, torularhodin). These biocomponents are widely used in food, pharmaceutical, cosmetic, chemical and fuel industries [9]-[11]. R. glutinis can accumulate approximately $40-70 \%$ of intracellular lipids of dry cell weight [12], [13]. This yeast primary synthesizes fatty acids like palmitic acid (C16:0), stearic acid (C18:0), oleic

*Corresponding author

E-mail address: Svetlana.raita@rtu.lv 
acid (C18:1), linoleic acid (C18:2) and linolenic acid (C18:3) [9], [14]. Lipids derived from yeasts has several advantages compared to vegetable and animal lipids. The yeast lipid production cycle is short, it has a rapid growth rate, and is independent of external conditions like climate and season [9].

Oleaginous yeasts are very efficient for cultivation on various low-cost waste substrates obtained from the agricultural, industrial and domestic sectors. They are able to consume carbon sources like sugars, glycerol, lactate, acetate and lipid-nature substrate [6], [15]. One of the most actively studied type of domestic waste as substrate for yeast production of addedvalue metabolites, is waste cooking oil (WCO) [16]. WCO is a vegetable oil-based substance consisting of edible vegetable matter that has been used for cooking at high temperatures and is no longer suitable for human consumption [15]. Oleaginous yeasts have catabolic and metabolic pathways for the degradation of lipids and their further assimilation into fatty acids, triacylglycerols and alkanes in yeast cellular capsule. The lipids are usually degraded into free fatty acids by yeast extracellular lipases and incorporated into the cells by active transporters or by simple diffusion depending on the concentration difference [6]. As shown by our previous study [17], WCO is an effective substrate for the cultivation of $Y$. lipolytica with high biomass yield results, which can significantly reduce the cost of fermentation [17].

Generally, WCO is used as co-substrate in the culture medium and not as the only carbon source for the production of microbial lipids, lipase, biosurfactants, erythritol, citric acid [18]. Most studies report that medium supplementation with WCO as a carbon source - increases yeast biomass, lipid accumulation and lipase production [15], [19], [20]. Usually, oil emulsification in a medium is performed with the addition of surfactants or by the ultrasonication method [6], [7], [15], [21]. An emulsifier helps to diminish the size of oil droplets and improves their miscibility in an aqueous broth making them easily assimilated by oleaginous yeast [6], [22]. It is being confirmed in the study by Patel and Matsaka [6] that ultra-sonicated oil droplets in WCO medium are the most effectively assimilated in C. curvatus cells, which resulted in higher lipid content in biomass. In anotherstudy by Saenge [23], media supplementation with emulsifier significantly increases the weight of biomass, lipid content and carotenoid production by $R$. glutinis. Usually, oil emulsification in water for broth or solid media, surfactants like polysorbates are used the most often [18].

Polysorbates are the class of emulsifiers, used in the cosmetic, pharmaceutical, food industry and as an additive in microbiological growth media to solubilize hydrophobic components in water-based solutions. They are nonionic surfactants derived from ethoxylated sorbitan, esterified with fatty acids [24]. There are polyoxyethylene sorbitan monolaurate (Tween 20), polyoxyethylene sorbitan palmitate (Tween 40), polyoxyethylene sorbitan monostearate (Tween 60) and polyoxyethylene sorbitan monooleate (Tween 80) [25]. Commonly used type of polysorbate in the studies is Tween 80 [20], [22], [26], [27], however, in recent years, other types have also been used for medium supplementation [21], [23], [28], [29]. According to the literature, the addition of polysorbate in medium can increase the filamentous fungi [22], [26], bacteria [28], [30] and yeast [8], [19], [31] enzymatic activity. According to the authors, this effect is explained by the fact that the surfactant can change cell membrane ultrastructure, enhancing the permeability of microorganism cells, expediting the transport of matters through the cell membranes, increasing the substrate entry effectiveness and enzyme secretion [20], [27], [32]. In the Zeng et al. study [22], the addition of $0.15 \%$ Tween 80 increased amylase activity of Penicillium simplicissimum by $104 \%$ [22]. However, opposite results were obtained in Fabiszewska et al. work [19], where oil medium supplementation with $1 \%$ of Tween 80 inhibited $Y$. lipolytica extracellular lipase activity, compared with oil medium without Tween 80 supplementation [19]. An interesting conclusion was made by Reese and Maguire [33], that surfactant has the greatest effect on 
the enzymatic activity increase in those types of microorganisms that has low enzymatic activity by their nature. However, the effect of adding surfactant is not so significant in microorganisms with initially high enzymatic activity [33].

The aim of the study was to examine the influence of the oil rich medium supplementation with Tween 20, 40 and 80 on $R$. glutinis growth. The objectives of this study were:

1. To determine the most suitable polysorbate type for the $R$. glutinis cultivation based on the value of biomass;

2. To establish, whether polysorbates have an inhibitory effect on $R$. glutinis, adding each kind of polysorbate at a concentration of $0.5 \%, 1-10 \%$;

3. To select the optimal concentration of a suitable polysorbate type for $R$. glutinis cultivation.

\section{MAterials AND Methodology}

\subsection{Microorganism and Materials}

Red yeast Rodotorula glutinis wild type was obtained from the Microbial Strain Collection of Latvia at the University of Latvia. Before the experiment, yeasts were stored at $4{ }^{\circ} \mathrm{C}$ on the plate. To reactivate the yeasts, 1 whole loop culture was added in standard yeast extract peptone glucose broth (YPG) and cultivated overnight to obtain the inoculum for the subsequent experiment.

Waste cooking rapeseed oil was obtained from the local bakery product manufacturer ('Lāči' Ltd.). Tween 20, 40 and 80 were purchased from Sigma-Aldrich.

\subsection{Medium Preparation and Cultivation Experiments}

Yeast extract peptone glucose modified broth medium (mYPG) contained glucose $14.2 \mathrm{~g} / \mathrm{L}$, yeast extract $10 \mathrm{~g} / \mathrm{L}$, peptone from meat $10 \mathrm{~g} / \mathrm{L}$, potassium dihydrogen phosphate $7 \mathrm{~g} / \mathrm{L}$, magnesium sulphate heptahydrate $1 \mathrm{~g} / \mathrm{L}$, sodium sulphate $2 \mathrm{~g} / \mathrm{L}, \mathrm{pH} 5.6$. Tween 20,40 and 80 with $0.5 \%, 1 \%, 2 \%, 3 \%, 4 \%, 5 \%, 6 \%, 7 \%, 8 \%, 9 \%, 10 \%$ concentrations each, were prepared and autoclaved along with $2 \%$ of waste cooking rapeseed oil in $50 \mathrm{~mL}$ Erlenmeyer flasks. Then in flasks was added autoclaved mYPG broth of $15 \mathrm{~mL}$ volume.

$R$. glutinis with an initial count of $1.0 \times 10^{4}$ cell $/ \mathrm{mL}$ broth was inoculated in the experimental flasks with three replicates and were incubated on a rotary shaker $(200 \mathrm{rpm})$ at $28^{\circ} \mathrm{C}$ for 7 days.

\subsection{Dry Biomass Measure}

Yeast biomass was characterized by dry biomass measured by the thermogravimetric method at $105^{\circ} \mathrm{C}$ and determined as gram per litre $(\mathrm{g} / \mathrm{L})$, measured dry weight every day during the $7^{\text {th }}$ days experiment. One millilitre of the culture has been taken for biomass weight measures at intervals of $24 \mathrm{~h}$. The culture was centrifuged at $10000 \times \mathrm{g}, 4{ }^{\circ} \mathrm{C}$ for $15 \mathrm{~min}$ and the supernatant was removed. Then wet biomass was washed with $0.5 \mathrm{~mL}$ hexane and centrifuged at $10000 \times \mathrm{g}, 4{ }^{\circ} \mathrm{C}$ for $15 \mathrm{~min}$ for removing oil from the biomass, and was dried at $105^{\circ} \mathrm{C} 24 \mathrm{~h}$ in a $1.5 \mathrm{~mL}$ centrifuge tube with an open cap.

\subsection{Statistical Analysis}

Represented data are the means of three replicates. Biomass value results depending of Tween type and its concentration were statistically analysed through two-way analysis of 
variance (ANOVA) with post hos Duncan multiple range test (IBM SPSS Statistics v26). Means with $\mathrm{p}$-values less than 0.05 were considered statistically significant.

\section{Results}

\subsection{Effect of Concentration $0.5 \%, 1 \%, 2 \%, 3 \%, 4 \%, 5 \%$ of Polysorbates on the R. glutinis Biomass}

In the first stage of the experiment, concentrations $0.5 \%, 1 \%, 2 \%, 3 \%, 4 \%$ and $5 \%$ were selected for each Tween 20, 40 and 80. R. glutinis dry biomass was measured for three types of polysorbates effect assessment on yeast growth during the 7 days of cultivation. Biomass growth of the $R$. glutinis was observed in the overall experiment cultivation period (Fig. 1), with the highest values on the $7^{\text {th }}$ day.
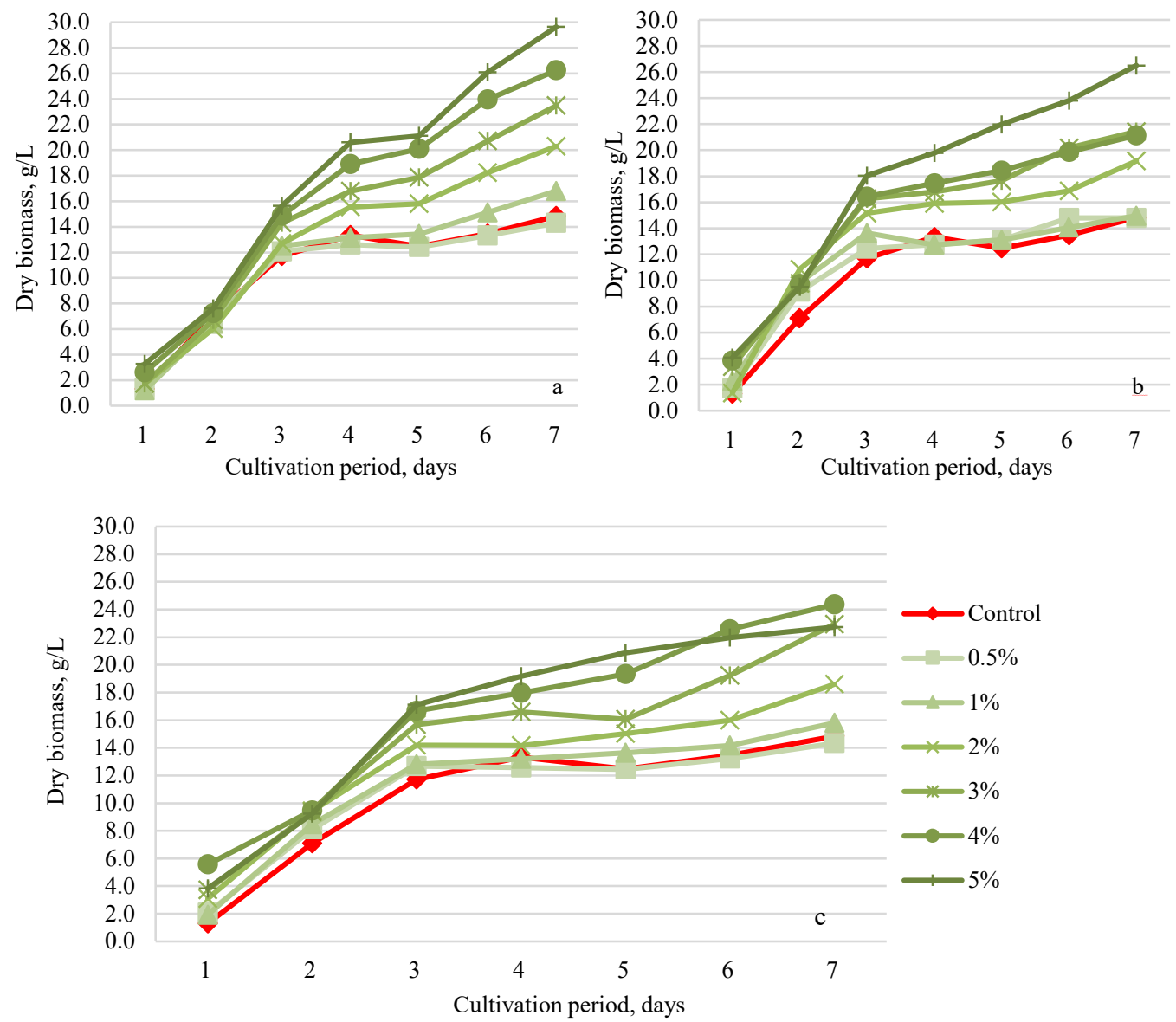

Fig. 1. R. glutinis biomass weight during cultivation period for samples with $0.5 \%, 1-5 \%$ concentration: a) Tween 20 ; b) Tween 40; c) Tween 80 supplementation compared with control without polysorbate. 
Dry biomass weight in the control flasks without added polysorbate on the $7^{\text {th }}$ day of cultivation was $14.83 \pm 0.93 \mathrm{~g} / \mathrm{L}$. Similar biomass weight results $14.30 \pm 0.72 \mathrm{~g} / \mathrm{L}$, $14.80 \pm 0.57 \mathrm{~g} / \mathrm{L}, 14.97 \pm 0.25 \mathrm{~g} / \mathrm{L}$ and $14.33 \pm 0.06 \mathrm{~g} / \mathrm{L}$, were obtained from the samples with $0.5 \%$ Tween $20,0.5 \%, 1 \%$ Tween 40 and $0.5 \%$ Tween 80 , respectively. Statistical analysis showed, that there was no statistically significant difference between the control and polysorbates concentrations $0.5 \%$ and $1 \%$ for each tween type $(p>0.05)$. With an increase in the concentration of polysorbates, the biomass of $R$. glutinis also increased. When $2 \%$ Tween 20, 40, and 80 were added to the medium, achieved biomass weights were $20.30 \pm 0.53 \mathrm{~g} / \mathrm{L}, 19.17 \pm 1.19 \mathrm{~g} / \mathrm{L}$, and $18.60 \pm 1.15 \mathrm{~g} / \mathrm{L}$, respectively. Addition of subsequent concentrations, the maximum biomass weight on the $7^{\text {th }}$ day of cultivation was $29.63 \pm 1.25 \mathrm{~g} / \mathrm{L}$ for $5 \%$ Tween $20,26.50 \pm 4.26 \mathrm{~g} / \mathrm{L}$ for $5 \%$ Tween 40 and $24.37 \pm 5.17 \mathrm{~g} / \mathrm{L}$ for Tween 80 at $4 \%$. The result for $5 \%$ Tween 80 showed biomass reduction of $22.73 \pm 3.36 \mathrm{~g} / \mathrm{L}$ compared to the $4 \%$, but these results showed also greater variation in weight between the three replicates, which is displayed in higher standard deviation (Fig. 2).

When considering the highest results of dry biomass weight of $R$. glutinis on the $7^{\text {th }}$ day of cultivation among the three Tween types, Tween 20 appeared to be the most effective. Although, two-way ANOVA test showed that there was no statistically significant difference between Tween types for concentrations from 0.5 to $5 \%(p>0.05)$. The effect of different concentration applications on biomass weight has been found to be statistically significant $(p<0.05)$. As shown by the Duncan test, there was a significant statistical difference between means of biomass weight on the $7^{\text {th }}$ day of cultivation as a function of polysorbates concentration (Fig. 2).



Fig. 2. R. glutinis dry biomass weight after 7 cultivation of days for control without Tween compared with supplementation of Tween 20 (T20), 40 (T40) and 80 (T80) with concentrations $0.5 \%, 1-5 \%$. The letters a, b, c, d represent a statistically significant differences between means of biomass weight on $7^{\text {th }}$ day of cultivation as a function of polysorbates concentration.

An interesting observation was obtained on the $5^{\text {th }}$ day of cultivation of $R$. glutinis. The dark orange substance appeared on the surface of the broth in the experimental samples with Tween 20, which was not observed in samples with Tween 40 and 80 (Fig. 3). It was presented in higher quantities in samples with $3 \%, 4 \%, 5 \%, 6 \% 7 \%$ of Tween 20 , and lesser in $0.5 \%$, $1 \%, 2 \%, 8 \%, 9 \%, 10 \%$. 




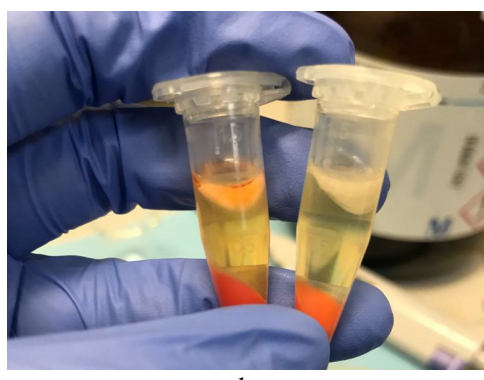

b

Fig. 3. Released dark orange substance on the surface of the broth in samples with Tween 20 at the concentration: a) $3 \%$, $4 \%, 5 \%$; b) $5 \%$ Tween 20 compared with $5 \%$ Tween 80 .
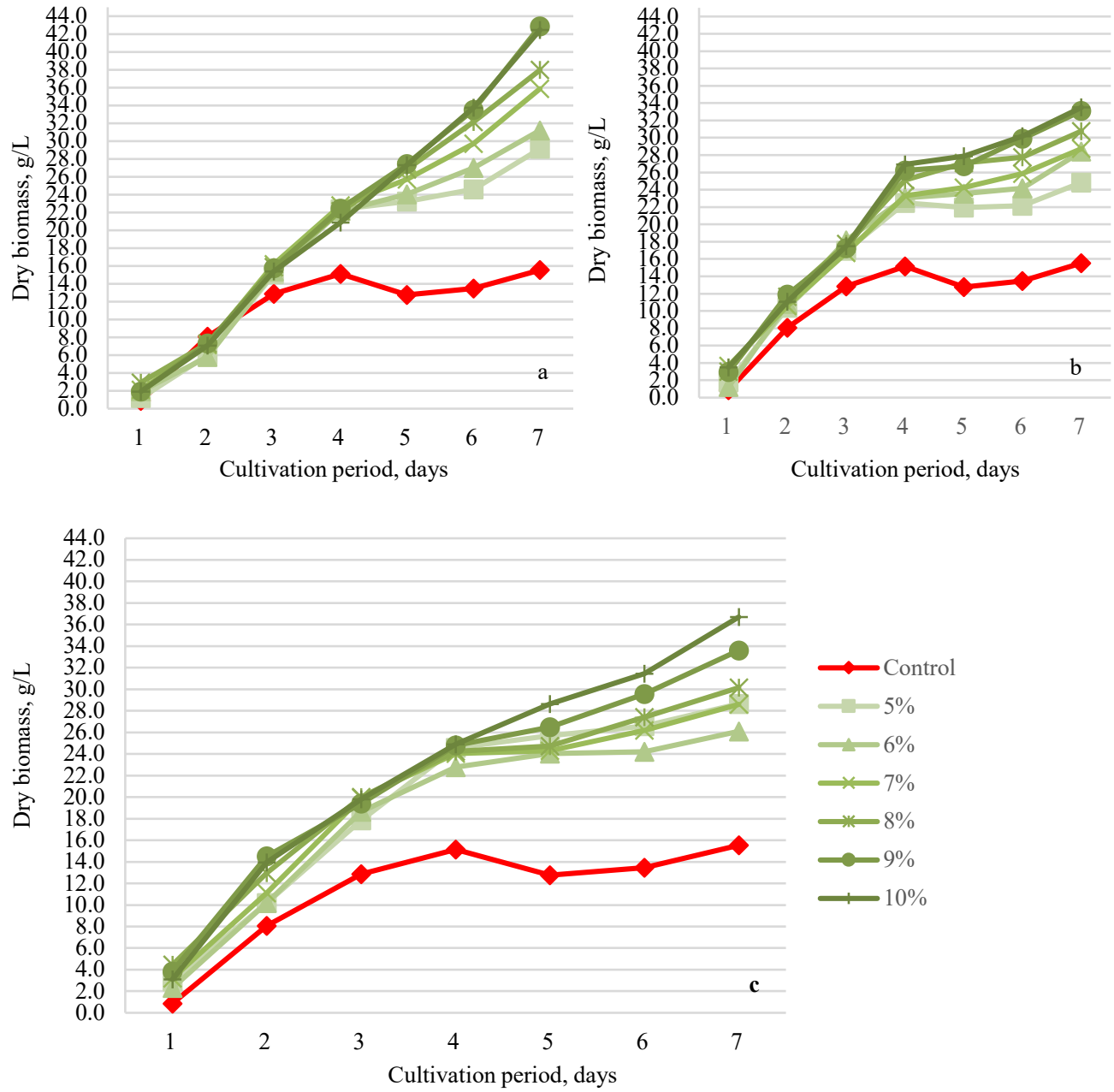

Fig. 4. R. glutinis biomass weight during cultivation period for samples with $5-10 \%$ concentration of: a) Tween 20 ; b) Tween 40; c) Tween 80 supplementation compared with control without polysorbate. 


\subsection{Effect of Concentration 5-10\% of Polysorbates on the R. glutinis Biomass}

In order to determine if polysorbates have an inhibitory effect on $R$. glutinis growth, the experiment was repeated with the addition of concentrations of $5 \%, 6 \%, 7 \%, 8 \%, 9 \%$ and $10 \%$ for each polysorbate type. For control, oil rich medium without polysorbate supplementation was used. The tendency of the biomass increase along with an increase in the concentration of Tweens continues even when polysorbates are added to the medium up to $10 \%$ (Fig. 4 ).

The addition of $9 \%$ and $10 \%$ to the medium had a similar result on the biomass of $R$. glutinis on the $7^{\text {th }}$ day of cultivation within the polysorbate types, i.e. $42.87 \pm 0.96 \mathrm{~g} / \mathrm{L}$ and $42.47 \pm 3.79 \mathrm{~g} / \mathrm{L}, 33.10 \pm 1.42$ and $33.50 \pm 0.80$ for Tween 20 and 40, respectively (Fig. 5). In the oil rich medium supplemented by Tween 80 , the weight of biomass has continued to increase - at $9 \%$ it was $33.60 \pm 2.97 \mathrm{~g} / \mathrm{L}$ and $36.70 \pm 1.41$ for $10 \%$ concentration. As shown by the Duncan test, there was no significant statistical difference between means of $R$. glutinis biomass weight on the $7^{\text {th }}$ day of cultivation as a function of $9 \%$ and $10 \%$ polysorbates concentration.

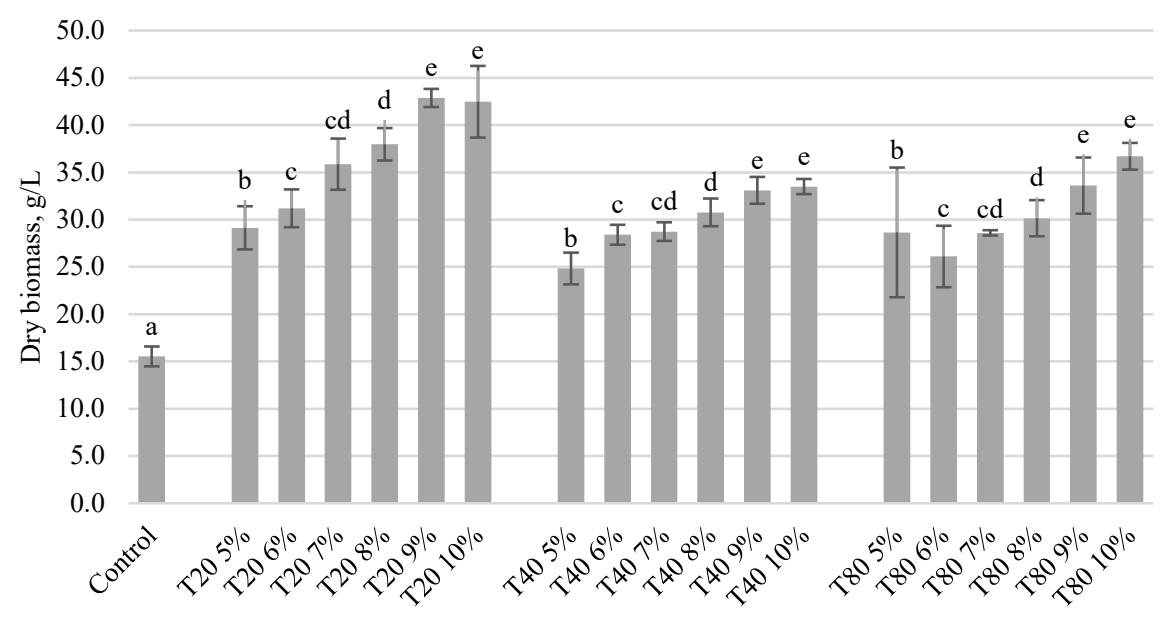

Fig. 5. R. glutinis biomass weight after 7 days of cultivation. The control sample without Tween is compared with the supplementation of Tween 20 (T20), 40 (T40) and 80 (T80) with concentrations 5-10\%. The letters a, b, c, d, e represent statistically significant differences between means of biomass weight on the $7^{\text {th }}$ day of cultivation, as a function of polysorbates concentration.

Unlike the lower polysorbate concentrations used in this study $(0.5 \%, 1 \%$ to $5 \%)$, Tween concentrations from $5 \%$ to $10 \%$ had statistically significant difference between Tween 20 and Tween 40, Tween 80 types $(p<0.05)$, which basically means, that the biomass weight output is not significantly affected by the type of used Tween, if its concentrations are $0.5-$ $5 \%$, while the concentrations $5 \%$ to $10 \%$ shows higher results with Tween 20 , than it is with Tween 40 and Tween 80 . The inhibitory effect of the growth of $R$. glutinis has not been observed when $0.5-10 \%$ of Tween 20,40 and 80 were added to the oil rich medium.

\section{Discussion}

In this study, the addition of polysorbates Tween 20,40 and 80 at concentrations ranging from $2 \%$ to $10 \%$ to the oil rich medium, significantly increased the biomass of $R$. glutinis 
(Fig. 1, Fig. 4). According to the literature, this positive effect of polysorbates on biomass growth can be explained in different ways.

Firstly, polysorbates have emulsifying properties reducing the size of oil droplets and improving their miscibility with aqueous broth, so that the oil is easily utilized by oleaginous yeast [5], [6], [21]. For example, Patel and Matsakas [6] have compared C. curvatus consumption of sonicated WCO with unsonicated, and found out, that after 72 hours of culture cultivation, $C$. curvatus was able to consume $98.75 \%$ and $73.85 \%$ sonicated and unsonicated WCO accordingly, and described the observation by the oil droplet size difference [6]

Secondly, possible reason for the increase in biomass weight may be associated with the ability of some microorganisms to use polysorbates as a carbon source. It was found that some microorganisms as microalgae Tetradesmus obliquus [34] and Lactobacillus plantarum [30] can utilize the fatty acid moieties of polysorbates successfully in the biosynthetic pathways. In the current study, the addition of the $10 \%$ Tween 20,40 and 80 showed the highest results and have increased biomass weight by $186 \%, 126 \%$ and $147 \%$ respectively, compared to the control (Fig. 5). One would assume that R. glutinis can utilize polysorbate as a carbon source, which would explain such high biomass growth. A similar degree of increase in the microorganism biomass has not been shown in previous works. Generally, the concentration of polysorbates used in the studies is limited to $0.2-0.5 \%$, which is necessary for the highest microbial lipids production and enzymatic activity, but this concentration is not able to increase the biomass yield [5], [15], [35]. Furthermore, some studies have shown that the polysorbate addition to the medium has less significance in yeast biomass weight growth than it is for the other carbon sources [19], [36]. In the study of yeast $Y$. lipolytica cultivation by Fabiszewska et al. [19], use of Tween $802 \%$ and $4 \%$ had a lesser effect on the biomass increase compared to the medium where only oil, oil with $1 \%$ Tween or glucose with oil as carbon sources were used. The other study [36] has reported that the use of $1.6 \%$ of Tween 20,40 and 80 as the only carbon source for cultivation of filamentous fungi Mortierella alpina increased lipid accumulation, but biomass weight was significantly lower compared with the glucose, i.e. $0.3 \pm 0.2 \mathrm{~g} / \mathrm{L}, 0.6 \pm 0.2 \mathrm{~g} / \mathrm{L}, 0.7 \pm 0.5 \mathrm{~g} / \mathrm{L}$ and $3.1 \pm 0.5 \mathrm{~g} / \mathrm{L}$, respectively [36]. In this regard, the assumption that the high weight of biomass in this study is associated with the use of polysorbates as a carbon source has no clear justification.

Thirdly, according to the literature, polysorbate at optimal concentration increases the permeability of microorganism cells, expediting the transport of matters through the cell membranes, resulting in the increase in substrate entry effectiveness and enzyme secretion. On the other hand, the effect of polysorbates on the permeability of the cell membrane of a microorganism depends on the concentration and can have both positive and negative effects [30], [34]. Esakkimuthu et al. [34] study shows that the addition of $0.1 \%$ polysorbates Tween 20,40 , and 80 to the medium increases the growth of the biomass of microalgae T. obliquus; with an increase in concentration to $0.15 \%$, the weight of the biomass decreased. When $0.2 \%$ polysorbates were added, the growth of the microalgae completely retorted. This polysorbate inhibitory effect is described by the mechanism of interfering with cellular membrane porosity and therefore, leading to cell damage [34]. In the current study, polysorbate Tween 20, 40 and 80 did not inhibit $R$. glutinis growth at concentrations up to $10 \%$. As our experiment shows, the optimal concentration for $R$. glutinis cultivation can be $2 \%$, since adding $0.5 \%$ and $1 \%$ of three types of polysorbates did not affect the biomass, but the selection of the higher concentrations is not cost-effective. However, polysorbate addition of concentrations above $2 \%$ may be considered if it has a positive effect on value-added products such as carotenoids.

Tween 80 is the most often described polysorbate type in the studies [20], [22], [26], [27]. In the studies where several types of polysorbates or all 4 are tested, the selection of the 
preferred type of polysorbate depends on the species of cultured microorganism. In the current study, the preferred polysorbate type for $R$. glutinis cultivation was Tween 20 . This polysorbate had a greater effect on biomass weight (Fig. 2, Fig. 5), and also stimulated the production of the dark orange substance, presumably carotenoids (Fig. 3). However, further research is needed to accurately determine the substance. Similar results were obtained in the Saenge et al. study [23], where Tween 20 was found to be the preferred polysorbate for the biomass increase, as well as the increase in lipid content and carotenoid production of $R$. glutinis TISTR 5159, compared to the control (without polysorbate) and Tween 80 supplementation in broth, containing palm oil mill effluent. The authors reported maximal biomass weight on the third day of cultivation. The results were $6.29 \pm 0.16 \mathrm{~g} / \mathrm{L}, 7.07 \pm 0.23 \mathrm{~g} / \mathrm{L}$ and $6.64 \pm 0.28 \mathrm{~g} / \mathrm{L}$ for control, $1 \%$ Tween 20 and $1 \%$ Tween 80 , respectively [23]. However, in our case, the third cultivation day of cultivation did not give the maximum biomass yield. We cannot declare the effectiveness of the polysorbate types based on 3-day results, since during the 4-7 days of $R$. glutinis cultivation, the growth trend lines were levelled and the difference between the concentrations became visible more clearly.

In this study, the addition of polysorbates 40 and 80 in the rapeseed oil rich medium had an equal effect on the $R$. glutinis growth. Although these two types of polysorbates had the least effect on the biomass weight compared with Tween 20, they still can be used for $R$. glutinis cultivation. In other studies, the preferable polysorbate for microorganism cultivation was Tween 80. Authors [32] reported that Tween 80 compared with Tween 20 affected the growth of $Y$. lipolytica cultivation in palm oil mill effluent more positively [32]. In the other study, [30] reported that the preferable polysorbate for the growth of $L$. plantarum RPR42 in various cane molasses-based media was Tween 80 followed by Tween 20, 60 and 40, respectively [30]. As some studies show, the type of oil added to the medium is an important factor. In the Agrawal study [21], the preferable polysorbate type for isolation and enumeration of lipophilic yeast Malassezia furfur was Tween 80 compared with Tween 20, 40 and 60 for 5 different oil types. Between Tween 20 and 40, the highest colony count was obtained from Tween 40 supplemented in olive and sesame oils solid medium; for palm, corn and coconut oil solid medium, Tween 40 and 20 had not got significant differences. Tween 60 showed fewer results between all polysorbate types for all mentioned types of oil [21].

This study had several limitations. The experiment lasted for 7 days, while the growth of $R$. glutinis biomass continued after the $7^{\text {th }}$ day. It is necessary to extend the cultivation period until the stationary phase and the death phase of $R$. glutinis growth could be observed. It was also impossible to thoroughly emulsify the oil into the broth due to the small volume of the experimental medium. For future prospects, ultrasonification or other emulsification techniques should be employed to emulsify the oil into a mediumat small volumes with and without polysorbates addition.

\section{Conclusion}

In this study, the addition of the polysorbates Tween 20,40 and 80 at concentrations ranging from $2 \%$ to $10 \%$ to the oil rich medium, has significantly increased the biomass of R. glutinis. All three types of the studied polysorbate concentrations of $0.5 \%$ and $1 \%$ did not affect the growth of the yeast and the dry biomass weight results were similar to the control without polysorbate supplementation. Despite the fact that there was no significant statistical difference between the three types of polysorbates at concentrations from $0.5 \%$ to $5 \%$ based on the biomass weight on the $7^{\text {th }}$ day of cultivation, Tween 20 was selected as the preferable polysorbate for $R$. glutinis cultivation. The optimal Tween 20 concentration for $R$. glutinis cultivation was $2 \%$, since adding $0.5 \%$ and $1 \%$ of three types of polysorbates did not affect 
the biomass yield, and the selection of higher concentration is not economically reasonable. Cultivation of $R$. glutinis in waste cooking rapeseed oil rich medium with polysorbates Tween 20,40 and 80 supplementations up to $10 \%$ of concentration does not have an inhibitory effect on the biomass growth. An interesting improvement of the study for the future prospects would be implied ultra-sonification or other emulsification techniques for better oil emulsification into the broth in cases of small volumes. As well as for the analysis of $R$. glutinis growth, it is necessary to extend the cultivation period until the stationary and death phases are observed.

\section{ACKNOWLEDGEMENT}

The work has been supported by ERDF project KC-PI-2020/31 "Utilization of biodegradable by-products into Protein Rich Extract for Either Ruminants or fish - Single Cell Protein" managed by the Investment and Development Agency of Latvia (LIAA).

\section{REFERENCES}

[1] Steele D. B., Stowers M. D. Techniques for selection of industrially important microorganisms. Annual Review of Microbiology 1991:45:89-106. https://doi.org/10.1146/annurev.mi.45.100191.000513

[2] Rapp P., Backhaus S. Formation of extracellular lipases by filamentous fungi, yeasts, and bacteria. Enzyme and Microbial Technology 1992:14(11):938-943. https://doi.org/10.1016/0141-0229(92)90059-W

[3] Fernández-Reiriz M. J., et al. Biomass production and variation in the biochemical profile (total protein, carbohydrates, RNA, lipids and fatty acids) of seven species of marine microalgae. Aquaculture 1989:83(1-2):17-37. https://doi.org/10.1016/0044-8486(89)90057-4

[4] Shen H., et al. Kinetics of continuous cultivation of the oleaginous yeast Rhodosporidium toruloides. Journal of Biotechnology 2013:168(1):85-89. https://doi.org/10.1016/j.jbiotec.2013.08.010

[5] Huang C., et al. Combined "de novo" and "ex novo" lipid fermentation in a mix-medium of corncob acid hydrolysate and soybean oil by Trichosporon dermatis. Biotechnology for Biofuels 2017:10:147. https://doi.org/10.1186/s13068$\underline{017-0835-8}$

[6] Patel A., Matsakas L. A comparative study on de novo and ex novo lipid fermentation by oleaginous yeast using glucose and sonicated waste cooking oil. Ultrasonics Sonochemistry 2019:52:364-374. https://doi.org/10.1016/j.ultsonch.2018.12.010

[7] Lopes M., et al. Waste Cooking Oils as Feedstock for Lipase and Lipid-Rich Biomass Production. European Journal of Lipid Science and Technology 2019:121(1):1-9. https://doi.org/10.1002/ejlt.201800188

[8] Pi H. W., et al. Engineering the oleaginous red yeast Rhodotorula glutinis for simultaneous $\beta$-carotene and cellulase production. Scientific Reports 2018:8:2-11. https://doi.org/10.1038/s41598-018-29194-Z

[9] Kot A. M., et al. Effect of initial $\mathrm{pH}$ of medium with potato wastewater and glycerol on protein, lipid and carotenoid biosynthesis by Rhodotorula glutinis. Electronic Journal of Biotechnology 2017:27:25-31. https://doi.org/10.1016/i.ejbt.2017.01.007

[10] Kuan I. C., et al. Microbial biodiesel production by direct transesterification of Rhodotorula glutinis biomass. Energies 2018:11(5):1036. https://doi.org/10.3390/en11051036

[11] Mussagy C. U., et al. Improvement of carotenoids production from Rhodotorula glutinis CCT-2186. Biochemical Engineering Journal 2021:165:107827. https://doi.org/10.1016/j.bej.2020.107827

[12] Elfeky N., et al. Lipid and carotenoid production by Rhodotorula glutinis with a combined cultivation mode of nitrogen, sulfur, and aluminium stress. Applied Sciences (Switzerland) 2019:9(12):2444. https://doi.org/10.3390/app9122444

[13] Maza D. D., et al. Growth and lipid production of Rhodotorula glutinis R4, in comparison to other oleaginous yeasts. Journal of Biotechnology 2020:310:21-31. https://doi.org/10.1016/j.jbiotec.2020.01.012

[14] Schneider T., et al. Lipid and carotenoid production by oleaginous red yeast Rhodotorula glutinis cultivated on brewery effluents. Energy 2013:61:34-43. https://doi.org/10.1016/j.energy.2012.12.026

[15] Yen H. W., Hu C. Y., Liang W. S. A cost efficient way to obtain lipid accumulation in the oleaginous yeast Rhodotorula glutinis using supplemental waste cooking oils (WCO). Journal of the Taiwan Institute of Chemical Engineers 2019:97:80-87. https://doi.org/10.1016/j.jtice.2019.02.012

[16] De Feo G., et al. Evolution of waste cooking oil collection in an area with long-standing waste management problems. Sustainability (Switzerland) 2020:12(20):8578. https://doi.org/10.3390/su12208578

[17] Spalvins K., et al. Waste Cooking Oil as Substrate for Single Cell Protein Production by Yeast Yarrowia lipolytica. Environmental and Climate Technologies 2020:24:457-469. https://doi.org/10.2478/rtuect-2020-0116 
[18] Lopes M., et al. Microbial valorization of waste cooking oils for valuable compounds production-a review. Critical Reviews in Environmental Science and Technology 2020:50(24):2583-2616. https://doi.org/10.1080/10643389.2019.1704602

[19] Fabiszewska A. U., et al. Carbon source impact on Yarrowia lipolytica KKP 379 lipase production. Applied Biochemistry and Microbiology 2014:50:404-410. https://doi.org/10.1134/S000368381404005X

[20] Rakicka M., et al. Enhanced production of erythritol and mannitol by Yarrowia lipolytica in media containing surfactants. Brazilian Journal of Microbiology 2016:47:417-423. https://doi.org/10.1016/j.bjm.2016.01.011

[21] Agrawal V., Bhagwat A. M., Sawant C. S. Sesame oil incorporated medium for isolation and enumeration of lipophilic yeasts. International Journal of Pharmaceutical Sciences and Research 2004:5. https://doi.org/10.13040/IJPSR.09758232.5(7).2972-79

[22] Zeng G. M., et al. Effects of Tween 80 and rhamnolipid on the extracellular enzymes of Penicillium simplicissimum isolated from compost. Enzyme and Microbial Technology 2006:39(7):1451-1456. https://doi.org/10.1016/j.enzmictec.2006.03.035

[23] Saenge C., et al. Efficient concomitant production of lipids and carotenoids by oleaginous red yeast Rhodotorula glutinis cultured in palm oil mill effluent and application of lipids for biodiesel production. Biotechnology and Bioprocess Engineering 2011:16:23-33. https://doi.org/10.1007/s12257-010-0083-2

[24] Rodríguez-López L., et al. Biological surfactants vs. polysorbates: Comparison of their emulsifier and surfactant properties. Tenside, Surfactants, Detergents 2018:55(4):273-280. https://doi.org/10.3139/113.110574

[25] Sannaningannavar F. M., et al. Ultrasonic study of thermo-acoustic parameters of the polysorbate 20, 40, 60 and 80 liquid surfactants at different temperatures. Journal of Molecular Liquids 2014:196:244-248. https://doi.org/10.1016/j.molliq.2014.03.039

[26] Nemec T., Jernejc K. Influence of Tween 80 on lipid metabolism of an Aspergillus niger strain. Applied Biochemistry and Biotechnology - Part A Enzyme Engineering and Biotechnology 2002:101:229-238. https://doi.org/10.1385/ABAB:101:3:229

[27] Wen Q., et al. Improved performance of air-cathode microbial fuel cell through additional Tween 80. Journal of Power Sources 2011:196(3):899-904. https://doi.org/10.1016/j.jpowsour.2010.09.009

[28] Reitermayer D., et al. Interrelation between Tween and the membrane properties and high pressure tolerance of Lactobacillus plantarum. BMC Microbiology 2018:18:72. https://doi.org/10.1186/s12866-018-1203-y

[29] Hsieh C., et al. Effect of plant oil and surfactant on the production of mycelial biomass and polysaccharides in submerged culture of Grifola frondosa. Biochemical Engineering Journal 2008:38(2):198-205. https://doi.org/10.1016/j.bej.2007.07.001

[30] Papizadeh M., et al. Using Various Approaches of Design of Experiments for High Cell Density Production of the Functionally Probiotic Lactobacillus plantarum Strain RPR42 in a Cane Molasses-based Medium. Current Microbiology 2020:77:1756-1766. https://doi.org/10.1007/s00284-020-01979-4

[31] Ciafardini G., Zullo B. A. Effect of lipolytic activity of Candida adriatica, Candida diddensiae and Yamadazyma terventina on the acidity of extra-virgin olive oil with a different polyphenol and water content. Food Microbiology 2015:47:12-20. https://doi.org/10.1016/j.fm.2014.10.010

[32] Louhasakul Y., et al. Enhanced valorization of industrial wastes for biodiesel feedstocks and biocatalyst by lipolytic oleaginous yeast and biosurfactant-producing bacteria. International Biodeterioration and Biodegradation 2020:148:104911. https://doi.org/10.1016/j.ibiod.2020.104911

[33] Reese E. T., Maguire A. Surfactants as stimulants of enzyme production by microorganisms. Applied microbiology 1969:17:242-245. https://doi.org/10.1128/aem.17.2.242-245.1969

[34] Esakkimuthu S., et al. Simultaneous induction of biomass and lipid production in Tetradesmus obliquus BPL16 through polysorbate supplementation. Renewable Energy 2019:140:807-815. https://doi.org/10.1016/j.renene.2019.03.104

[35] Xu J., et al. Renewable microbial lipid production from Oleaginous Yeast: some surfactants greatly improved lipid production of Rhodosporidium toruloides. World Journal of Microbiology and Biotechnology 2016:32:107. https://doi.org/10.1007/s11274-016-2076-6.

[36] Wynn J. P., Ratledge C. Evidence that the rate-limiting step for the biosynthesis of arachidonic acid in Mortierella alpina is at the level of the 18:3 to 20:3 elongase. Microbiology 2000:146:2325-2331. https://doi.org/10.1099/00221287-146-9-2325 